\title{
Direct photon production from hadronic sources in high-energy heavy-ion collisions
}

\author{
E.L. Bratkovskaya, ${ }^{1}$ S.M. Kiselev, ${ }^{2}$ and G.B. Sharkov ${ }^{2}$ \\ ${ }^{1}$ Frankfurt Institute for Advanced Studies, Frankfurt, Germany \\ ${ }^{2}$ Institute for Theoretical and Experimental Physics, Moscow, Russia
}

\begin{abstract}
The low $p_{T}$ direct photon production from a variety of the hadronic sources is studied within the microscopic HSD transport approach for $p+C, p+P b$ and $P b+P b$ collisions at $160 \mathrm{~A} \mathrm{GeV}$. The direct photon emission from elementary hadronic scatterings as well as meson-meson bremsstrahlung are incorporated. The influence of in-medium effects such as a collisional broadening of the vector-meson spectral functions on the photon emission rate is found to be hardly observable in the final spectra which are dominated by bremsstrahlung type processes. The uncertainties in the subtraction of the 'background' from the photon decay of hadronic resonances inside the hot and dense fireball is investigated, additionally. Our findings are relevant for the interpretation and extraction of experimental data on direct photon production at low $p_{T}$.

PACS numbers: 25.75-q, 25.75.Cj, 24.10.Lx
\end{abstract}




\section{INTRODUCTION}

The properties of hadronic matter under extreme densities and temperatures and their phase transition to the deconfined and strongly interacting Quark-Gluon-Plasma (QGP) are the central topics of modern high-energy physics. In order to understand the dynamics and relevant scales of this transition laboratory experiments under controlled conditions are presently performed with ultra-relativistic nucleus-nucleus collisions. The electromagnetic radiation (real and virtual photons, i.e. dilepton pairs) is the unique probe to study the heavy-ion collisions since the photons do not suffer from the final state interactions with the surrounding matter and, thus, provide a clear signal of the various environments of their creations (cf. the pioneering works [1, 2, 3]). Moreover, the photons are emitted from all stages of the collisions - from the QGP to the hadronic phase, so they may provide information about the different degrees of freedom - from partons to hadrons [4, 5, 6, 6].

On the quark-gluon level the main processes for the direct photon production are Compton scattering of quarks and gluons $q g \rightarrow q \gamma$ and annihilation of quark and anti-quarks $q \bar{q} \rightarrow g \gamma$ as leading processes, while the next leading order (NLO) process is dominated by bremsstrahlung $q g \rightarrow q g \gamma$. The photons radiated from the different sources have a different transverse momentum $p_{T}$ that allows to separate the contributions: The photons with high $p_{T}$ are primarily produced by the initial hard NN collisions and called as 'prompt' (or 'hard') photons. Direct

photons from a thermalised QGP dominate at lower transwerse momentum $\left(1 \leq p_{T} \leq 3 \mathrm{GeV} / \mathrm{c}\right)$ and denoted as 'thermal photons'. On the hadronic level the main source of direct photons is meson-meson rescattering: $\pi \rho \rightarrow \pi \gamma, \pi \pi \rightarrow \rho \gamma, \pi K \rightarrow K^{*} \gamma, K \rho \rightarrow K \gamma, \ldots$, where the first two channels are the most important. These photons from the hadronic rescattering dominate at even lower $p_{T}$. Additionally there are photons coming from the hadron decays ( $\operatorname{such}$ as $\pi^{0}, \eta$ etc.), which give a huge photonic 'background' and make the experimental measurement of direct photons very complicated.

Most of the theoretical predictions for direct photons are based on the local thermalization assumption and evaluate the photon production rates from the equilibrated quark-gluon plasma or hadronic matter which then are convoluted with the space-time evolution of the system. In order to account for the non-equilibrium dynamics with its full complexity one needs microscopic transport models. However, most of the transport models - commonly used for the description 
of high energy heavy-ion collisions - are based on the hadron-string picture (e.g. HSD $^{1}$ and $\mathrm{UrQMD}^{2}$ ) and do not include the phase transition from partonic to hadronic matter in a consistent way, nor solve the hadronization problem. We mention that there are attempts to develop such transport models, e.g. the multi-phase transport model (AMPT) [8] which includes pQCD-like partonic scattering, or combined models - hydrodynamics for the QGP + UrQMD for the hadronic stage [9], or Parton-Hadron-String-Dynamics (PHSD) model which is based on a dynamical quasiparticle model matched to reproduce lattice QCD results in thermodynamic equilibrium [10].

We recall that already in 1995-1997 there were attempts do describe the first experimental photon data by WA98 Collaboration on $\mathrm{S}+\mathrm{Au}$ at $200 \mathrm{~A} \mathrm{GeV}$ [11] in the transport models - UrQMD [12], AMPT [13] and HSD [14]. The transport calculations included the photon production by the hadronic decays [12, 13, 14] as well as direct photon production from the meson-meson scattering [12, 13]. Also the influence of in-medium effects such as dropping vector meson masses on the photon yield have been addressed in Refs. [13, 14]. Unfortunately, the first WA98 data provided only an upper bound for the direct photon production yield and did not allow to draw solid conclusions - all transport results were just below the upper limit given by WA98. In 2000 the WA98 Collaboration provided new data for $\mathrm{Pb}+\mathrm{Pb}$ at $160 \mathrm{~A} \mathrm{GeV}$ [15]. This stimulated a new wave of interest for direct photons from the theoretical side (cf. the review [16]) and references therein).

The aim of our present study is to investigate the photon production at SPS energies from hadronic sources using the extended version of the HSD transport model [17, 18] which includes the off-shell dynamics of the vector mesons with dynamical spectral functions. It allows to study the influence of in-medium effects - such as a collisional broadening - on the photon emission rate. We will compare our transport calculations to the experimental data for $\mathrm{Pb}+\mathrm{Pb}$ at $160 \mathrm{~A}$ $\mathrm{GeV}$ as well as to the new preliminary data on photon production in $\mathrm{p}+\mathrm{C}$ and $\mathrm{p}+\mathrm{Pb}$ collisions at $160 \mathrm{~A} \mathrm{GeV} \mathrm{[19].} \mathrm{We} \mathrm{stress} \mathrm{here} \mathrm{again} \mathrm{that} \mathrm{the} \mathrm{HSD} \mathrm{model} \mathrm{doesn't} \mathrm{include} \mathrm{the} \mathrm{phase} \mathrm{transition}$ from QGP to hadronic matter; we thus concentrate on the low $p_{T}$ photon production which is dominated by hadronic sourses. First 'pilot' HSD results have been reported in Ref. [20].

Our paper is organized as follows: In Section II we describe the treatment of various channels

1 Hadron-String-Dynamics transport approach

${ }^{2}$ Ultra-relativistic-Quantum-Molecular-Dynamics 
for the photon production in HSD. Section III contains a comparison of the HSD results with the WA98 data for $\mathrm{Pb}+\mathrm{Pb}$ at $160 \mathrm{~A} \mathrm{GeV}$, while Section IV contains our results for photon production in $\mathrm{p}+\mathrm{C}$ and $\mathrm{p}+\mathrm{Pb}$ collisions at $160 \mathrm{~A} \mathrm{GeV}$. A summary closes this work in Section V.

\section{PHOTON PRODUCTION IN HSD}

Our analysis is carried out within the HSD transport model [14, 21, 22] - based on covariant self energies for the baryons [23] - that has been used for the description of $p A$ and $A A$ collisions from SIS to RHIC energies. We recall that in the HSD approach nucleons, $\Delta^{\prime}$ 's, $\mathrm{N}^{*}(1440)$, $\mathrm{N}^{*}(1535), \Lambda, \Sigma$ and $\Sigma^{*}$ hyperons, $\Xi$ 's, $\Xi^{*}$ 's and $\Omega$ 's as well as their antiparticles are included on the baryonic side whereas the $0^{-}$and $1^{-}$octet states are incorporated in the mesonic sector. Inelastic baryon-baryon (and meson-baryon) collisions with energies above $\sqrt{s}_{t h} \simeq 2.6 \mathrm{GeV}$ (and $\sqrt{s_{t h}} \simeq 2.3 \mathrm{GeV}$ ) are described by the FRITIOF string model [24] whereas low energy hadron-hadron collisions are modeled in line with experimental cross sections. Note that the HSD transport approach includes the off-shell dynamics of vector mesons explicitly; for the details we address the reader to Ref. [17].

We consider the following hadronic sources of photon production:

I. The photon production by a mesonic decays $\left(\pi^{0}, \eta, \eta^{\prime}, \omega, \phi, a_{1}\right)$ where the mesons are produced firstly in baryon-baryon $(B B)$, meson-baryon $(m B)$ or meson-meson $(m m)$ collisions. The photon production from the mesonic decays represents a 'background' for the search of the direct photons, however, this background is very large relative to the expected direct photon signal. Moreover, there are a severe experimental difficulties in subtracting the photons from hadronic decays. For the present study we consider the contributions from the photon decay of the following mesons:

$$
\begin{aligned}
& \pi^{0} \rightarrow \gamma+\gamma, \\
& \eta \rightarrow \gamma+\gamma, \\
& \eta^{\prime} \rightarrow \rho+\gamma, \\
& \omega \rightarrow \pi^{0}+\gamma, \\
& \phi \rightarrow \eta+\gamma, \\
& a_{1} \rightarrow \pi+\gamma .
\end{aligned}
$$


The decay probability is calculated according to the corresponding branching ratios taken from PDG [25]. The broad resonances in the initial/final state are treated in line with to their in-medium spectral functions which will be illustrated in the next subsections.

II. Additionally to the resonance/meson decay channels the photons can be produced directly in elementary collisions of particles. For the present study we consider the direct photon production by the scattering processes

$$
\begin{aligned}
& \pi \pi \rightarrow \rho \gamma \\
& \pi \rho \rightarrow \pi \gamma
\end{aligned}
$$

accounting for all possible charge combinations. We note, that we discard $\gamma$-production in rescattering processes with strange mesons (such as $\pi+K^{*} \rightarrow K+\gamma, \pi+K \rightarrow K^{*}+\gamma, K+K^{*} \rightarrow$ $\pi+\gamma$ etc.), since at SPS energies the strange meson density is subdominant. However, as indicated in Ref. [26] such processes may play a role at higher energies. In the next subsections we will define the contributions of the processes $\pi \pi \rightarrow \rho \gamma, \pi \rho \rightarrow \pi \gamma$ explicitly.

III. The photon production from meson-meson bremsstrahlung might play an important role, too, as has been pointed out firstly by Haglin [27] and later on by Liu and Rapp [28]. These authors have calculated the thermal photon emission rate from the proceses $m+m \rightarrow m+m+\gamma$ and found that the bremsstrahlung radiation is one of the dominant channels for low energy photon production.

In the next subsections we define explicitly the treatment of the dominant hadronic process for direct photon production.

\section{A. Direct photon production in $\pi \pi \rightarrow \rho \gamma$ reactions}

Since at SPS energies the pion density is relatively high, the $\pi \pi$ annihilation channel has to be accounted for in the direct photon production. With increasing energies (and pion densities) the importance of this channel grows accordingly.

The basic form of the cross section for the $\pi \pi \rightarrow \rho \gamma$ reaction has been adopted from Kapusta et al. in Ref. [29] where the high-energy photon production has been evaluated in the hot hadron gas model. However, in Ref. [29] the final $\rho$ meson has been considered on-shell, i.e. at the pole mass $M_{0}=0.77 \mathrm{GeV}$. Since in HSD the vector mesons are treated with the full off-shell spectral function, which depends on density and momentum [17], some modification of 
the formulae from Ref. [29] has to be done in order to account for the broad mass distribution of the final $\rho$ meson. The simplest way is to 'fold' the cross section from Ref. [29] with the mass and density dependent spectral function of the $\rho$-mesons.

We note, that for the present study we use the same form of spectral functions for the vector mesons as modeled in Ref. [17]. In particular, the spectral function of a vector meson $V$ with mass $M$ at nucleon density $\rho_{N}$ is taken in the Breit-Wigner form:

$$
A_{V}\left(M, \rho_{N}\right)=C_{1} \cdot \frac{2}{\pi} \frac{M^{2} \Gamma_{V}^{*}\left(M, \rho_{N}\right)}{\left(M^{2}-M_{0}^{*^{2}}\left(\rho_{N}\right)\right)^{2}+\left(M \Gamma_{V}^{*}\left(M, \rho_{N}\right)\right)^{2}},
$$

with the normalization condition for any $\rho_{N}$ :

$$
\int_{M_{m i n}}^{M_{\text {lim }}} A_{V}\left(M, \rho_{N}\right) d M=1
$$

where $M_{\text {lim }}=2 \mathrm{GeV}$ is chosen as an upper limit for the numerical integration. The lower limit of the vacuum $\rho$ spectral function corresponds to the $2 \pi$ decay $M_{\min }=2 m_{\pi}$, whereas for the in-medium collisional broadening case $M_{\text {min }}=2 m_{e} \rightarrow 0$ with $m_{e}$ denoting the electron mass. $M_{0}^{*}$ is the pole mass of the vector meson spectral function which is $M_{0}^{*}\left(\rho_{N}=0\right)=M_{0}$ in vacuum, however, shifted in the medium for the dropping mass scenario according to the Hatsuda and Lee [30] or Brown/Rho scaling [31]. Futhermore, the vector meson width has been implemented as:

$$
\Gamma_{V}^{*}\left(M,|\vec{p}|, \rho_{N}\right)=\Gamma_{V}(M)+\Gamma_{\text {coll }}\left(M,|\vec{p}|, \rho_{N}\right)
$$

Here $\Gamma_{V}(M)$ is the total width of the vector mesons $(V=\rho, \omega)$ in the vacuum. For the $\rho$ meson we use

$$
\begin{gathered}
\Gamma_{\rho}(M) \simeq \Gamma_{\rho \rightarrow \pi \pi}(M)=\Gamma_{0}\left(\frac{M_{0}}{M}\right)^{2}\left(\frac{q}{q_{0}}\right)^{3} F(M) \\
q=\frac{\left(M^{2}-4 m_{\pi}^{2}\right)^{1 / 2}}{2}, \quad q_{0}=\frac{\left(M_{0}^{2}-4 m_{\pi}^{2}\right)^{1 / 2}}{2}
\end{gathered}
$$

In (17) $\Gamma_{0}$ is the vacuum width of the $\rho$-meson at the pole mass $M_{0} ; F(M)$ is a form factor taken from Ref. [32] as

$$
F(M)=\left(\frac{2 \Lambda^{2}+M_{0}^{2}}{2 \Lambda^{2}+M^{2}}\right)^{2}
$$

with a cut-off parameter $\Lambda=3.1 \mathrm{GeV}$. This form factor was introduced in Ref. [32] in order to describe the $e^{+} e^{-}$experimental data. For the $\omega$ meson a constant total vacuum width is used: $\Gamma_{\omega} \equiv \Gamma_{\omega}\left(M_{0}\right)$, since the $\omega$ is a narrow resonance in vacuum. 
The collisional width in (6) is approximated as

$$
\Gamma_{\text {coll }}\left(M,|\vec{p}|, \rho_{N}\right)=\gamma \rho_{N}<v \sigma_{V N}^{t o t}>\approx \alpha_{c o l l} \frac{\rho_{N}}{\rho_{0}}
$$

Here $v=|\vec{p}| / E ; \vec{p}, E$ are the velocity, 3-momentum and energy of the vector meson in the rest frame of the nucleon current and $\gamma^{2}=1 /\left(1-v^{2}\right)$. Furthermore, $\sigma_{V N}^{t o t}$ is the meson-nucleon total cross section.

As discussed in Ref. [17] - in order to simplify the actual calculations - the coefficient $\alpha_{\text {coll }}$ has been extracted in the HSD transport calculations from the vector-meson collision rate in $A+A$ reactions as a function of the density $\rho_{N}$. The numerical results for $\Gamma_{\text {coll }}\left(\rho_{N}\right)$ then have been divided by $\rho_{N} / \rho_{0}$ to fix the coefficient $\alpha_{\text {coll }}$ in (9). We obtain $\alpha_{\text {coll }} \approx 150 \mathrm{MeV}$ for the $\rho$ and $\alpha_{\text {coll }} \approx 70 \mathrm{MeV}$ for $\omega$ mesons which are consistent with the experimental analysis in Ref. [33]. In this way the average effects of collisional broadening are incorporated and allow for an explicit representation of the vector-meson spectral functions versus the nuclear density, $\rho_{N}$.

We mention that also finite temperature effects lead to a sizable broadening of the vector mesons spectral functions (also at baryon chemical potential $\mu_{B}=0$ ). This is essentially due to scattering with mesons which may contribute to the total width by $70-80 \mathrm{MeV}$ at a temperature of $\sim 170 \mathrm{MeV}$ according to the early work by Haglin [34]. But for the present study we use a simplified modeling of the collisional broadening width which discard an explicit consideration of such temperature effects. However, the temperature effects are partly accounted here due to explicit meson-meson interactions which also lead to changes in the vector meson mass distribution. Since we find that the spectral broadening of the mesons is practically not visible in the final photon spectra, an explicit consideration of temperature effects is beyond the scope of this study.

In order to explore the observable consequences of vector meson mass shifts at finite nuclear density - as indicated by the CBELSA-TAPS data [35] for the $\omega$ meson - the in-medium vector meson pole masses are modeled (optionally) according to the Hatsuda and Lee [30] or Brown/Rho scaling [31] as

$$
M_{0}^{*}\left(\rho_{N}\right)=\frac{M_{0}}{\left(1+\alpha \rho_{N} / \rho_{0}\right)},
$$

where $\rho_{N}$ is the nuclear density at the resonance decay position $\vec{r}, \rho_{0}=0.16 \mathrm{fm}^{-3}$ is the normal nuclear density and $\alpha \simeq 0.16$ for the $\rho$ and $\alpha \simeq 0.12$ for the $\omega$ meson [33]. The parametrization (10) may be employed also at much higher collision energies (e.g. FAIR and SPS) and one does 

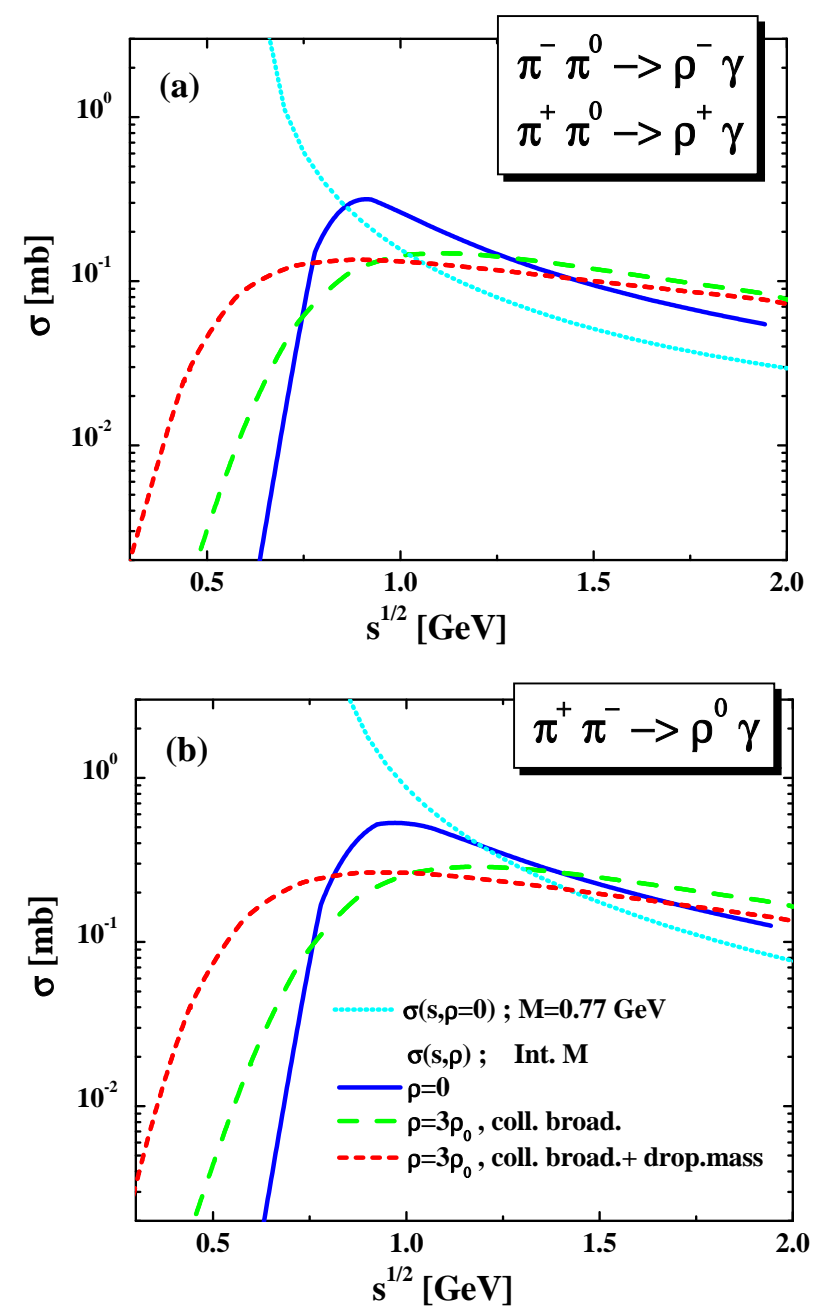

FIG. 1: (Color online) The $\gamma$-production cross section $\sigma(s, \rho)$ for the $\pi^{-}+\pi^{0} \rightarrow \rho^{-}+\gamma, \pi^{+}+\pi^{0} \rightarrow \rho^{+}+\gamma$ (upper part - (a)) and $\pi^{+}+\pi^{-} \rightarrow \rho^{0}+\gamma$ (lower part - (b)) reaction for $\rho_{N}=0$ (solid lines) and in the case of the 'collisional broadening' scenario (dashed lines) and the 'dropping mass + collisional broadening' scenario (short dashed lines) for nuclear density $\rho_{N}=3 \rho_{0}$. The dotted lines correspond to the results from Kapusta et al. 29] for the fixed $\rho$-meson mass $M=0.77 \mathrm{GeV}$, while all other lines show the yield integrated over the $\rho$-meson mass $M$.

not have to introduce a cut-off density in order to avoid negative pole masses. Note that (10) is uniquely fixed by the 'customary' expression $M_{0}^{*}\left(\rho_{N}\right) \approx M_{0}\left(1-\alpha \rho_{N} / \rho_{0}\right)$ in the low density regime.

Thus, we model the photon- $\rho$-meson production cross section in $\pi \pi$ reactions in the following 
way: The total cross section $\sigma_{\pi \pi \rightarrow \rho \gamma}\left(s, \rho_{N}\right)$ is

$$
\sigma_{\pi \pi \rightarrow \rho \gamma}\left(s, \rho_{N}\right)=\int_{M_{\min }}^{M_{\max }} d M \frac{d \sigma_{\pi \pi \rightarrow \rho \gamma}\left(s, M, \rho_{N}\right)}{d M} .
$$

The mass differential cross section is approximated by

$$
\frac{d \sigma_{\pi \pi \rightarrow \rho \gamma}\left(s, M, \rho_{N}\right)}{d M}=\sigma_{\pi \pi \rightarrow \rho \gamma}^{0}(s, M) \cdot A\left(M, \rho_{N}\right) \cdot \frac{\int_{M_{\min }}^{M_{\max }} A\left(M, \rho_{N}\right) d M}{\int_{M_{\min }}^{M_{\text {lim }}} A\left(M, \rho_{N}\right) d M},
$$

where $A\left(M, \rho_{N}\right)$ denotes the meson spectral function (4) for given total width $\Gamma_{V}^{*}$ (6); $M_{\max }=\sqrt{s}$ is the maximal kinematically allowed invariant mass of the $\rho$ meson. In Eq.

(12) $\sigma_{\pi \pi \rho \gamma}^{0}(s, M)$ is the vacuum cross section from Kapusta et al. [29] where the $\rho$-meson mass is considered as a free variable (i.e. not fixed to $0.77 \mathrm{GeV}$ as in [29]). Thus, formula (12) can be used to model the vector meson production in $\pi \pi$ reactions in the vacuum and in the medium, too.

Fig. 1 shows the $\gamma$-production cross section $\sigma(s, \rho)$ for the $\pi^{-}+\pi^{0} \rightarrow \rho^{-}+\gamma, \pi^{+}+\pi^{0} \rightarrow \rho^{+}+\gamma$ (upper part) and $\pi^{+}+\pi^{-} \rightarrow \rho^{0}+\gamma$ (lower part) reaction for $\rho_{N}=0$ (solid lines) and in case of the 'collisional broadening' scenario (dashed lines) and the 'dropping mass + collisional broadening' scenario (short dashed lines) for nuclear density $\rho_{N}=3 \rho_{0}$. The dotted lines correspond to the original cross section from Kapusta et al. [29] for the fixed $\rho$-meson mass $M=0.77 \mathrm{GeV}$, while all other lines show the cross section integrated over the mass $M$. Note that the cross section from Kapusta et al. [29] for the fixed $\rho$-meson mass diverges at threshold. However, the folding over the spectral function of the $\rho$ meson leads to an endothermic behavior of the cross section (cf. the solid line) instead of an exothermic (dotted line).

As seen from Fig. 1 the cross section in the 'collisional broadening' scenario is basically smeared out close to threshold. Only when incorporating additionally a dropping mass the thresholds are shifted down in energy such that the production cross sections become enhanced in the subthreshold regime with increasing nuclear density $\rho_{N}$.

\section{B. Direct photon production in $\pi \rho \rightarrow \pi \gamma$ reactions}

In Fig. 2 we show the production cross section $\sigma(s)$ for the $\pi^{-}+\rho^{0} \rightarrow \pi^{-}+\gamma, \pi^{+}+\rho^{0} \rightarrow \pi^{+}+\gamma$ (upper part), $\pi^{+}+\rho^{-} \rightarrow \pi^{0}+\gamma, \pi^{-}+\rho^{+} \rightarrow \pi^{0}+\gamma$ (middle part) and $\pi^{0}+\rho^{-} \rightarrow \pi^{-}+\gamma$, 

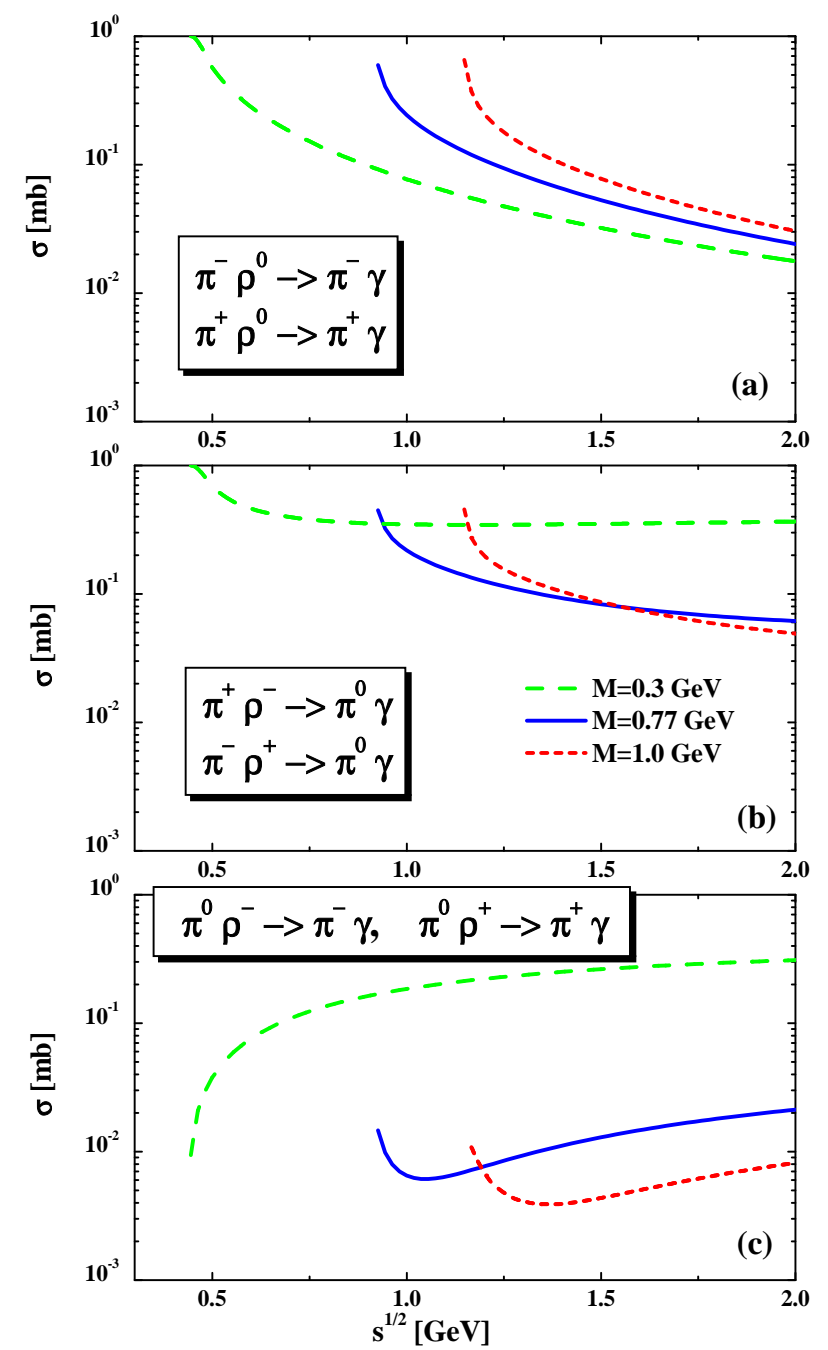

FIG. 2: (Color online) The production cross section $\sigma(s)$ for the $\pi^{-}+\rho^{0} \rightarrow \pi^{-}+\gamma, \pi^{+}+\rho^{0} \rightarrow \pi^{+}+\gamma$ (upper part - (a)), $\pi^{+}+\rho^{-} \rightarrow \pi^{0}+\gamma, \pi^{-}+\rho^{+} \rightarrow \pi^{0}+\gamma\left(\right.$ middle part - (b)) and $\pi^{0}+\rho^{-} \rightarrow \pi^{-}+\gamma$, $\pi^{0}+\rho^{+} \rightarrow \pi^{+}+\gamma$ (lower part - (c)) reaction. The dashed lines corresponds to the $\rho$-meson mass $M=0.3 \mathrm{GeV}$, the solid lines stand for $M=0.77 \mathrm{GeV}$ while the short dashed lines correspond to $M=1.0 \mathrm{GeV}$.

$\pi^{0}+\rho^{+} \rightarrow \pi^{+}+\gamma$ (lower part) reactions. Here again we use the vacuum cross sections from Kapusta et al. [29] and consider the $\rho$-meson mass as a free variable since the production of the vector mesons in HSD is realized with respect to the spectral function $A\left(M, \rho_{N}\right)$. The dashed lines corresponds to the $\rho$-meson mass $M=0.3 \mathrm{GeV}$, the solid lines stand for $M=0.77 \mathrm{GeV}$, while the short dashed lines correspond to $M=1.0 \mathrm{GeV}$.

As follows from Fig. 2 the $\pi+\rho \rightarrow \pi+\gamma$ cross section depends very strongly on the mass 
of the initial $\rho$ meson - for low $M$ the threshold is shifted to low $\sqrt{s}$. Thus, one can expect an enhancement of $\gamma$ production in case of this channel for the in-medium scenarios due to the enhanced population of the low mass $\rho$ mesons.

\section{Photon production by the decay $a_{1} \rightarrow \pi \gamma$}

The photons can be also emitted from the decay $a_{1} \rightarrow \pi \gamma$. In spite that the branching ratio of this process is not well known experimentally and expected to be very small (we use

$\left.\operatorname{Br}\left(a_{1} \rightarrow \pi \gamma\right) \sim 1.5 \cdot 10^{-3}[36]\right)$, this contribution is also considered in our investigation. The study of the $a_{1}$ dynamics is an interesting problem itself since it is related to chiral symmetry restoration at high densities and temperatures as pointed out in Refs. [37, 38, 39, 40].

The production of $a_{1}$ mesons in HSD stems from $B B$ and $m B$ collisions via string excitation and decay at high energies and by $\pi+\rho \leftrightarrow a_{1}$ reactions at low energies. The mass of the $a_{1}$ meson is distributed according to the Breit-Wigner spectral function - Eq. (4) - with a mass $(\mu)$ and density $\left(\rho_{N}\right)$ dependent width $\Gamma_{a_{1}}^{*}$. The total $a_{1}$ width $\Gamma_{a_{1}}$ can be written as

$$
\Gamma_{a_{1}}^{*}\left(\mu,|\vec{p}|, \rho_{N}\right)=\Gamma_{a_{1}}\left(\mu, \rho_{N}\right)+\Gamma_{a_{1}: c o l l}\left(\mu,|\vec{p}|, \rho_{N}\right)
$$

where the collisional width is approximated by $\Gamma_{a_{1} \text { coll }}\left(\mu,|\vec{p}|, \rho_{N}\right) \simeq \alpha_{a_{1} \text { :coll }} \rho_{N} / \rho_{0}$ with $\alpha_{a_{1}: \text { coll }} \simeq$ $150 \mathrm{MeV}$.

Since the $a_{1}$ meson decays dominantly to $\pi+\rho$ [39] one has to take into account the mass distribution of the final $\rho$ meson $A\left(M, \rho_{N}\right)$ when calculating the width of the $a_{1}$ meson. Thus formula (7) for the $a_{1}$ width $\Gamma_{a_{1}}\left(\mu, \rho_{N}\right)$ has to be modified accordingly:

$$
\begin{gathered}
\Gamma_{a_{1}}\left(\mu, \rho_{N}\right)=\Gamma_{a_{1}}^{0}\left(\frac{\mu_{0}}{\mu}\right)^{2} \frac{\int_{M_{\min }}^{\mu-m_{\pi}} d M q^{3}(\mu, M) A\left(M, \rho_{N}\right)}{\int_{\mu_{0}-m_{\pi}}^{M_{m i n}} d M q_{0}^{3}\left(\mu_{0}, M\right) A\left(M, \rho_{N}\right)} \\
q(\mu, M)=\frac{\sqrt{\lambda\left(\mu, M, m_{\pi}\right)}}{2 \mu}, \quad q_{0}\left(\mu_{0}, M\right)=\frac{\sqrt{\lambda\left(\mu_{0}, M, m_{\pi}\right)}}{2 \mu_{0}} .
\end{gathered}
$$

Here $\lambda(x, y, z)=\left(x^{2}-(y-z)^{2}\right)\left(x^{2}-(y+z)^{2}\right)$ while $m_{\pi}$ is the pion mass; $\mu_{0}, \Gamma_{a_{1}}^{0}$ are the vacuum pole mass and width of the $a_{1}$ spectral function with $\Gamma_{a_{1}}^{0}$ taken as $400 \mathrm{MeV}$.

According to Eq. (14) the $a_{1}$ width depends on the $\rho$ meson spectral function $A\left(M, \rho_{N}\right)$ and, thus, is sensitive to the $\rho$ meson in-medium effects. We stress, that such a 'coupling' of 


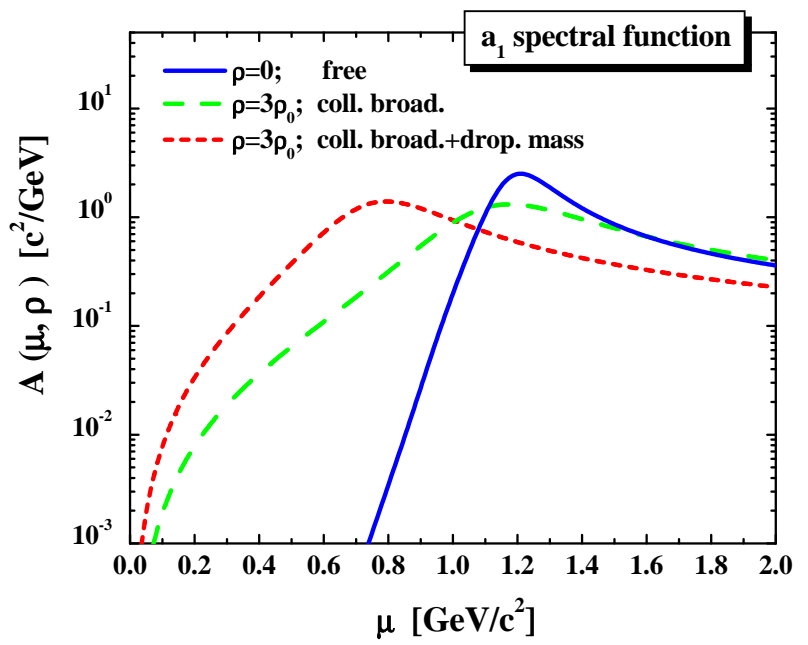

FIG. 3: (Color online) The spectral function for the $a_{1}$ meson for $\rho_{N}=0$ (solid line) and in case of the 'collisional broadening' scenario (dashed line) and the 'dropping mass + collisional broadening' scenario (short dashed line) for nuclear density $\rho_{N}=3 \rho_{0}$.

the $a_{1}$ and $\rho$ spectral functions has to be accounted for when drawing conclusions about an experimental observation of chiral symmetry restoration by measuring the $a_{1}$ meson properties in heavy-ion experiments (e.g. the corresponding results in Ref. [40] should be strongly effected).

Figure 3 shows the spectral function $A_{a_{1}}\left(\mu, \rho_{N}\right)$ for the $a_{1}$ meson for $\rho_{N}=0$ (solid line) and in the case of the 'collisional broadening' scenario (dashed line) and the 'dropping mass + collisional broadening' scenario (short dashed line) for nuclear density $\rho_{N}=3 \rho_{0}$. Here the in-medium scenarios are related to both the $a_{1}$ as well as the $\rho$ meson.

The production cross section $\sigma(s, M)$ for the $\pi+\rho \rightarrow a_{1}$ reaction (for all possible charge combinations) is calculated as follows,

$$
\sigma_{\pi+\rho \rightarrow a_{1}}\left(s, M, \rho_{N}\right)=\frac{6 \pi^{2}}{q^{2}(s, M)} \Gamma_{a_{1}}\left(s, \rho_{N}\right) A_{a_{1}}\left(s, \rho_{N}\right),
$$

where $s \equiv \mu^{2}$ and the width $\Gamma_{a_{1}}\left(s, \rho_{N}\right)$ and momentum $q(s, M)$ are defined by Eq. (14).

In Fig. 4 we display the production cross section $\sigma(s, M)$ for the $\pi+\rho \rightarrow a_{1}$ reaction for $\rho_{N}=0$ (solid lines) and in case of the 'collisional broadening' scenario (dashed lines) and the 'dropping mass + collisional broadening' scenario (short dashed lines) for nuclear density $\rho_{N}=3 \rho_{0}$. The upper part corresponds to the $\rho$ meson mass of $M=0.77 \mathrm{GeV}$ while the lower part shows the results for $M=0.3 \mathrm{GeV}$. As seen from Fig. 4 the $a_{1}$ production cross section in the $\pi+\rho$ reaction is very sensitive to the in-medium scenarios as well as to the mass $M$ of the initial $\rho$ meson (even in the free case - cf. solid lines). 

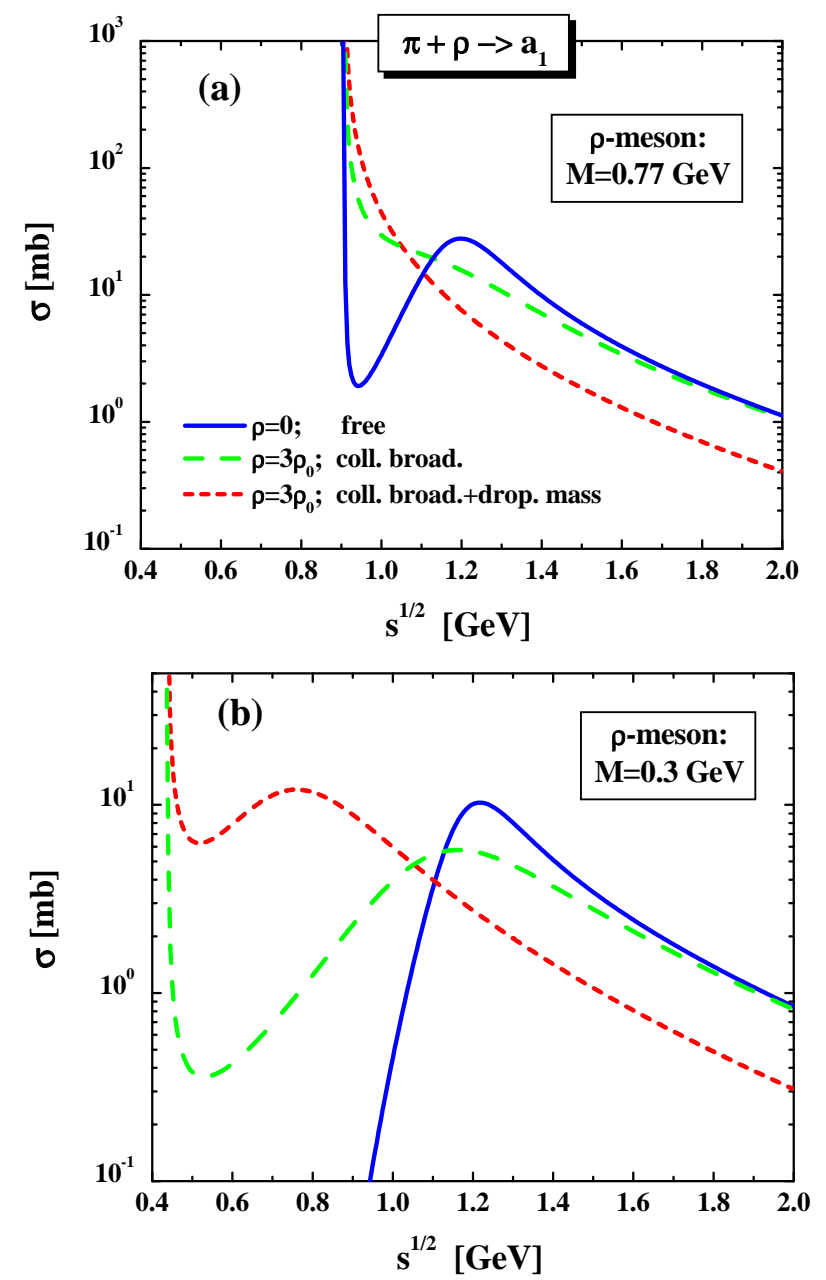

FIG. 4: (Color online) The production cross section $\sigma(s, M)$ for the $\pi+\rho \rightarrow a_{1}$ reaction for $\rho_{N}=0$ (solid lines) and in the case of the 'collisional broadening' scenario (dashed lines) and the 'dropping mass + collisional broadening' scenario (short dashed lines) for nuclear density $\rho_{N}=3 \rho_{0}$. The upper part (a) corresponds to $M=0.77 \mathrm{GeV}$ while the lower part (b) shows the results for $M=0.3 \mathrm{GeV}$.

\section{Direct photon production from meson-meson bremsstrahlung}

The implementation of photon bremsstrahlung from hadronic reactions in transport approaches is based on the 'soft photon' approximation. The soft-photon approximation (SPA) [41] relies on the assumption that the radiation from internal lines is negligible and the strong interaction vertex is on-shell. In this case the strong interaction part and the electromagnetic part can be separated, so the soft-photon cross section for the reaction $1+2 \rightarrow 1+2+\gamma$ can 
be written as

$$
\begin{aligned}
& q_{0} \frac{d^{3} \sigma^{\gamma}}{d^{3} q}=\frac{\alpha}{4 \pi} \frac{\bar{\sigma}(s)}{q_{0}^{2}} \\
& \bar{\sigma}(s)=\frac{s-\left(M_{1}+M_{2}\right)^{2}}{2 M_{1}^{2}} \sigma(s),
\end{aligned}
$$

where $M_{1}$ is the mass of the charged accelerated particle, $M_{2}$ is the mass of the second particle; $q_{0}, q$ are the energy and momentum of the photon. In (16) $\sigma(s)$ is the on-shell elastic cross section for the reaction $1+2 \rightarrow 1+2$. This approximation has also been employed by Haglin in Ref. [27].

In Ref. [28] the photon bremsstrahlung from $\pi+\pi$ and $\pi+K$ elastic collisions has been

calculated using the $U_{e m}(1)$-gauged meson-exchange model which includes the photon coupling to pseudoscalar and vector mesons. Indeed, such calculations go beyond the SPA model. However, the direct comparison of SPA and the $U_{\text {em }}(1)$ models - cf. Fig. 4 in Ref. [28] - show a very good agreement between the models, which allows us to use the simplified SPA formula for our purpose here. Thus, we have calculated the photon bremsstrahlung from all elastic meson-meson scattering $m_{1}+m_{2} \rightarrow m_{1}+m_{2}+\gamma\left(\right.$ where $\left.m=\pi, \eta, K, \bar{K}, K^{0}, K^{*}, \bar{K}^{*}, K^{* 0}\right)$, which occur during the heavy-ion collisions by applying the SPA formula (16)).

\section{RESULTS FOR THE PHOTON PRODUCTION IN A+A COLLISIONS AT SPS ENERGIES}

Now we step on to the description of direct photon production from heavy-ion collisions applying the HSD transport model incorporating all photon production channels from elementary reactions as described in the previous Section.

In Fig. 5 we start with a comparison of the HSD results for the inclusive photon transverse momentum distribution for $10 \%$ central $158 A \mathrm{GeV}{ }^{208} \mathrm{~Pb}+{ }^{208} \mathrm{~Pb}$ collisions with the experimental data from the WA98 Collaboration [15] (solid dots). We select photons in the pseudorapidity interval $2.35 \leq \eta \leq 2.95$ in the laboratory frame which corresponds to mid-rapidity in the center-of-mass frame. Our calculations show that such cut in pseudorapidity reduces the photon yield by about of factor 5 , however, practically doesn't change the shape of the $p_{T}$ spectra. The WA98 data correspond to the transverse momentum interval $0.5 \leq p_{T} \leq 4 \mathrm{GeV} / c$ while the HSD calculation includes also very low $p_{T}$ momenta but extends up to 2.5-3 GeV/c only since the high $p_{T}$ tail is very hardly reachable due to limitied statistics. 


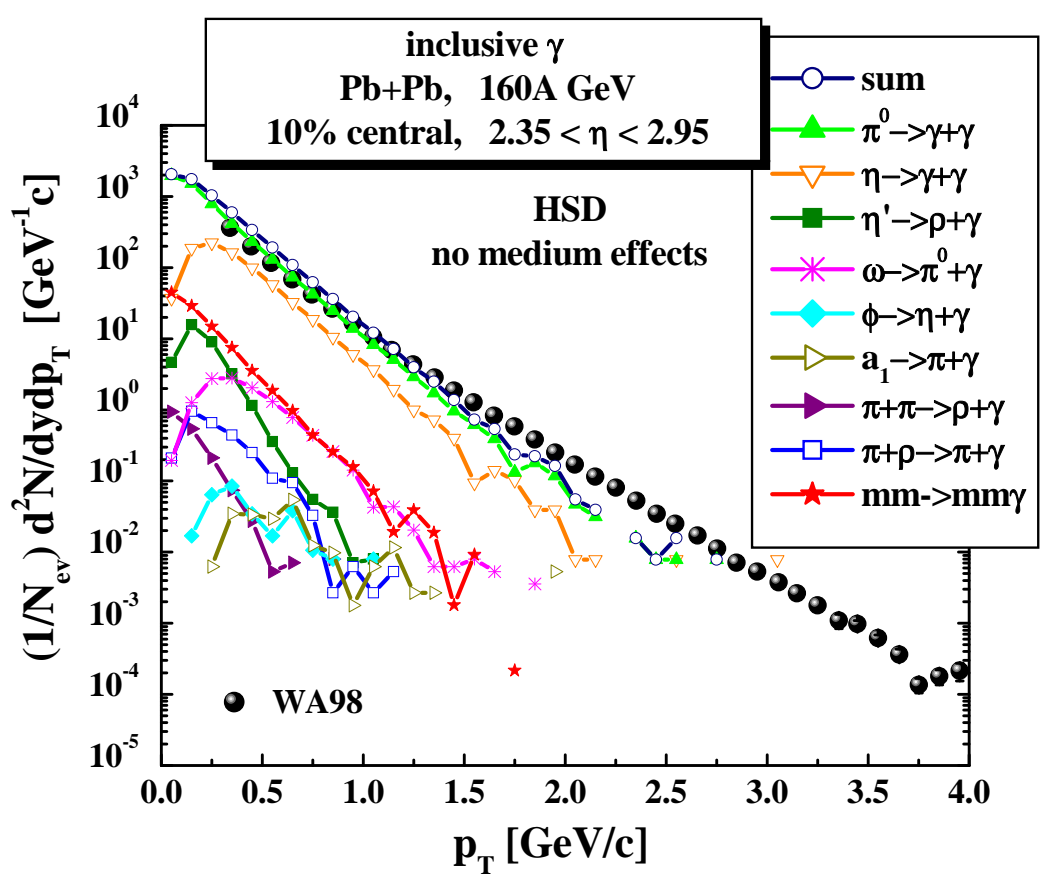

FIG. 5: (Color online) The inclusive photon transverse momentum distribution for central $158 A \mathrm{GeV}$ ${ }^{208} \mathrm{~Pb}+{ }^{208} \mathrm{~Pb}$ collisions at $2.35 \leq \eta \leq 2.95$. The solid symbols and arrows corresponds to the WA98 data [15], the lines correspond to the HSD results for the various channels as indicated in the legend (without medium effects for the vector mesons).

Fig. 5 presents the HSD calculations without medium effects for the vector mesons (the definition of the lines are indicated in the legend). As seen from Fig. 5 the dominant channels for the inclusive photon production are the photon from $\pi$ and $\eta$ decays $(\pi \rightarrow \gamma+\gamma$ and $\eta \rightarrow \gamma+\gamma$ ), whereas other channels are down by more than order of magnitude. The HSD results agree very well with the experimental data which is, indeed, expectable since the HSD model provides a good description of the pion transverse momentum spectra at SPS energies [42] and predicts a meson $m_{T}$ scaling [43].

In order to obtain the information about the direct photon production, one needs to subtract the 'background' contributions which are dominated by the mesonic decay processes. While the $\pi^{0}$ and $\eta$ spectra are measured directly by the same experiments [15], it is possible to estimate their photon decay in a reliable way. Indeed, the life times of $\pi$ 's and $\eta$ 's are large such that they basically decay at the end of reaction stage, i.e. in vacuum. However, the situation with the $\eta^{\prime} \rightarrow \rho+\gamma, \omega \rightarrow \pi^{0}+\gamma, \phi \rightarrow \eta+\gamma, a_{1} \rightarrow \pi+\gamma$ decays are not so transparent since these mesons cannot be mesured directly by the WA98 detector. 


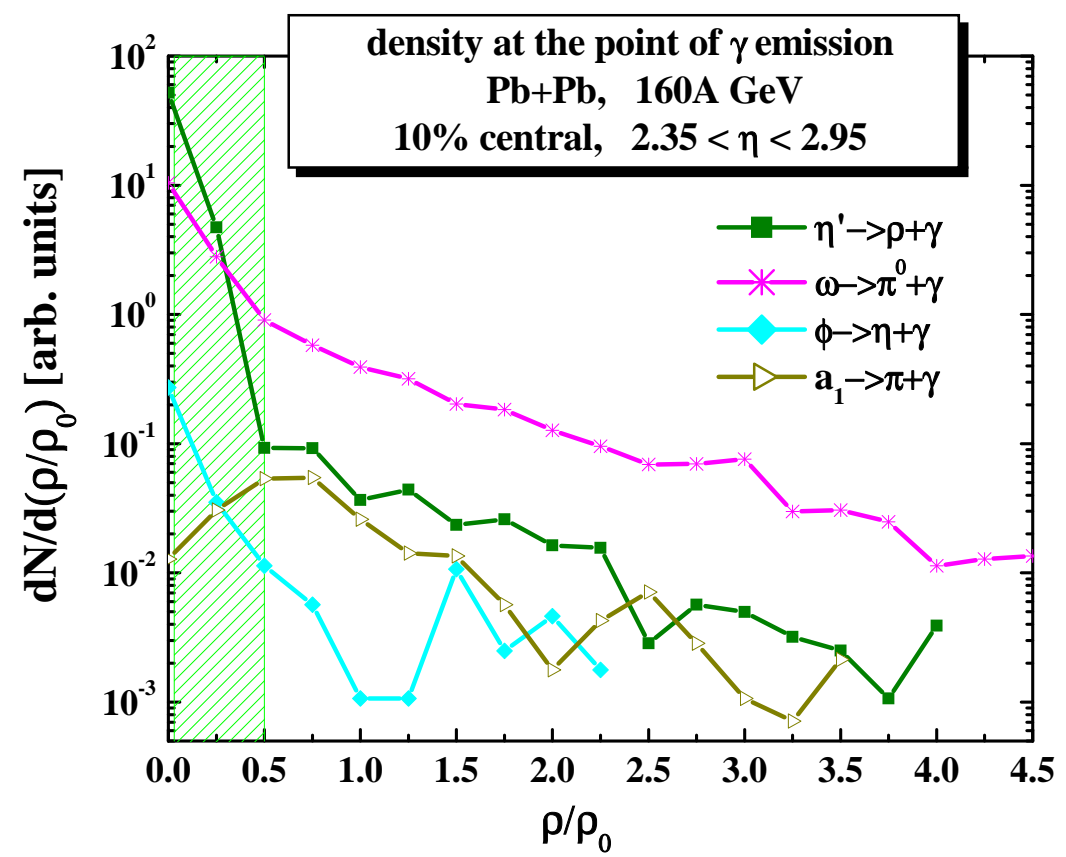

FIG. 6: (Color online) The nucleon density distribution at the photon emission point (in units of $\left.\rho_{0}\right)$ for central $158 A \mathrm{GeV}{ }^{208} \mathrm{~Pb}+{ }^{208} \mathrm{~Pb}$ collisions at $2.35 \leq \eta \leq 2.95$. The definition of the lines is indicated in the legend.

The WA98 Collaboration has subtracted the contribution of hadronic decays by calculations based on the assumption of $m_{T}$-scaling with the same slope of the $m_{T}$-spectra as measured in the $\pi^{0}$ spectrum and with relative normalizations $R_{\text {hadron } / \pi^{0}}$ (equivalent to the asymptotic ratios for $p_{T} \rightarrow \infty$ ) [15]. However, such a procedure is a rought approximation especially for the short living resonances which can emit photons inside the hot fireball shortly after creation and, thus, can not be reconstruct in experiment. This statement is illustrated in Fig. 6 which shows the density distribution at the photon emission points from $\eta^{\prime}, \omega, \phi, a_{1}$ decays for central $158 A \mathrm{GeV}{ }^{208} \mathrm{~Pb}+{ }^{208} \mathrm{~Pb}$ collisions at $2.35 \leq \eta \leq 2.95$. One can see from Fig. 6 that there is some fraction of $\omega, \phi, a_{1}$ mesons that emit photons for $\rho_{N} \geq \rho_{0} / 2$ (e.g. $\sim 25 \%$ of $\omega$ 's and $\sim 70 \%$ of $a_{1}$ 's), whereas a relatively long leaving $\eta^{\prime}$ meson decays dominantly at low densities.

In order to demonstrate the possible contribution of the in-medium decays of $\eta^{\prime}, \omega, \phi, a_{1}$ mesons to the direct photon spectra reported by the WA98 Collaboration we show in the r.h.s of Fig. 7 the invariant mid-rapidity spectra including the contribution of the $\eta^{\prime}, \omega, \phi, a_{1}$ photon decay only for $\rho_{N}>\rho_{0} / 2$ whereas the 1.h.s. of Fig. 7 shows the total contribution of all hadronic channels at all densities (for comparison). Indeed, such a cut in density leads to 

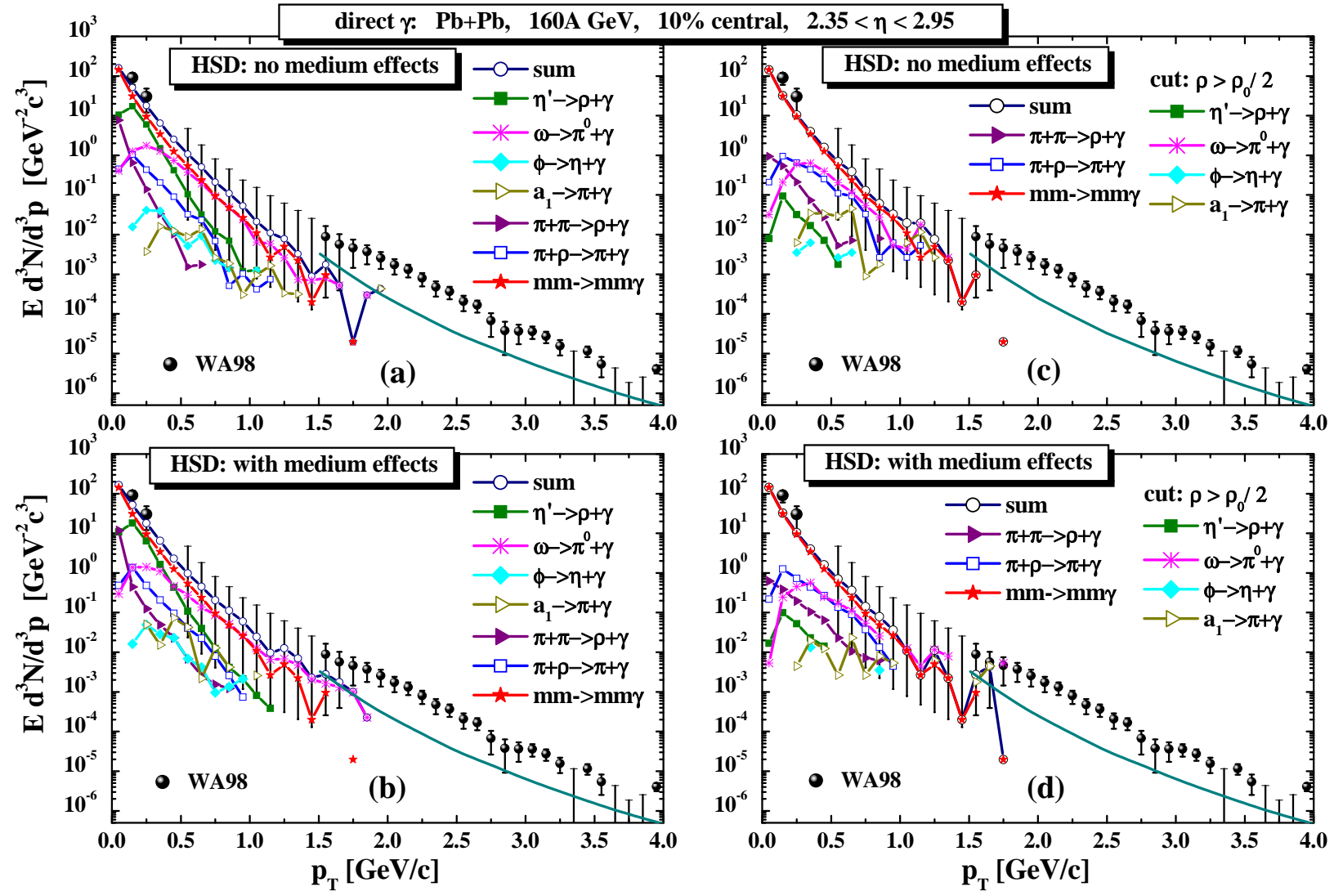

FIG. 7: (Color online) The invariant direct photon multiplicity for central $158 A \mathrm{GeV}{ }^{208} \mathrm{~Pb}+{ }^{208} \mathrm{~Pb}$ collisions at $2.35 \leq \eta \leq 2.95$. The solid symbols and arrows corresponds to the WA98 data [15], the lines stand for the HSD calculations without (upper row - (a),(c)) and with (lower row - (b),(d)) medium effects (i.e. collisional broadening) for the vector mesons. The definition of the lines is indicated in the legend. The solid line for $p_{T}>1.5 \mathrm{GeV} / c$ gives the contribution from the prompt photons. The right panel (c),(d) shows the spectra including the contribution of the $\eta^{\prime}, \omega, \phi, a_{1}$ photon decay only for $\rho_{N}>\rho_{0} / 2$ whereas the left panel (a),(b) shows the total contribution of all hadronic channels at all densities.

a substantial reduction of the hadronic decay 'background' especially for low $p_{T}$, however, it becomes essential at $p_{T} \leq 0.7 \mathrm{GeV} / \mathrm{c}$. Thus, our calculations provide the scale of possible uncertainties in the experimental background subtraction which is important for the physical interpretation of the experimental results.

One can see from Fig. 7 that the dominant channel for low $p_{T}$ photon production is mesonmeson bremsstrahlung. This is in line with the analysis by Haglin [27] and Liu and Rapp 


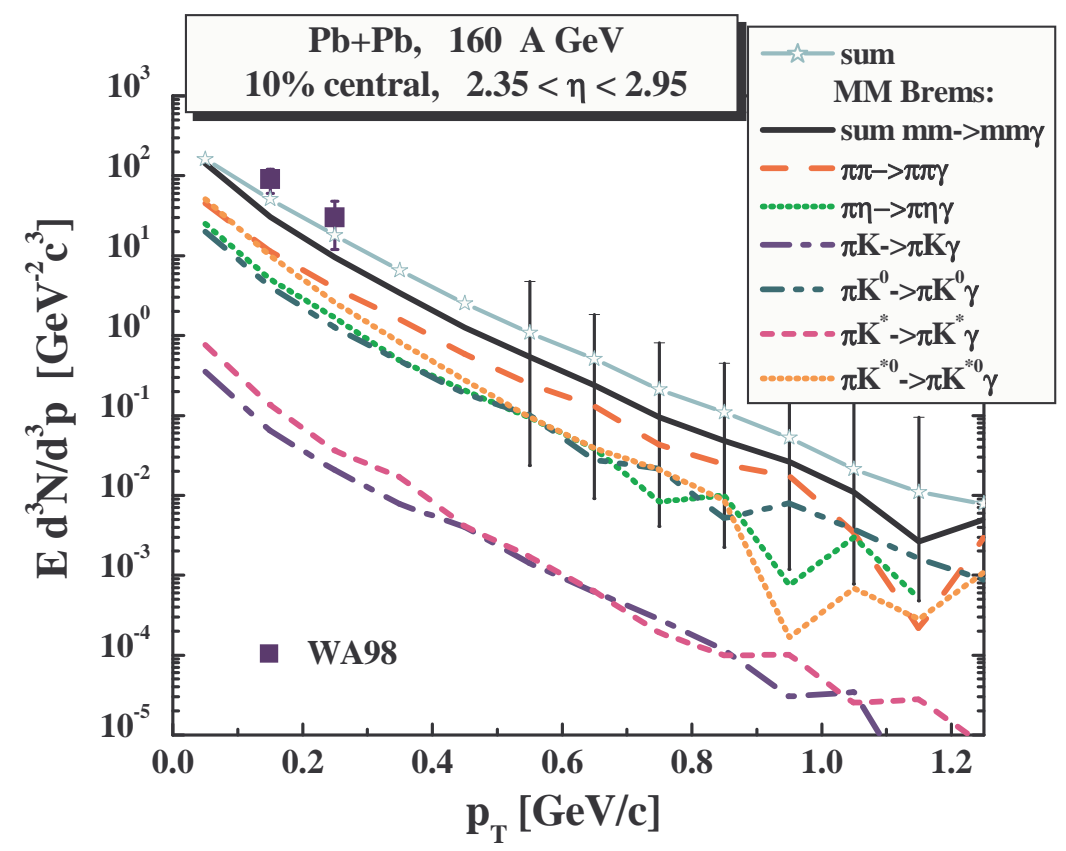

FIG. 8: (Color online) The invariant direct photon multiplicity for central $158 A \mathrm{GeV}{ }^{208} \mathrm{~Pb}+{ }^{208} \mathrm{~Pb}$ collisions at $2.35 \leq \eta \leq 2.95$. The solid symbols and arrows corresponds to the WA98 data [15], the lines stand for the HSD calculations: the upper solid line with the stars shows the sum over all channels, the solid line corresponds to the sum over all meson-meson bremsstrahlung channels which are specified by the various lines. The definition of the lines is indicated in the legend.

[28]. The contributions of the other channels such as direct photon production by mesonmeson collisions $\pi+\pi \rightarrow \rho+\gamma$ and $\pi+\rho \rightarrow \pi+\gamma$ are found to be suppressed relative to the meson-meson bremsstrahlung. Also we have investigated the influence of in-medium effects such as collisional broadening for the vector meson spectral functions which leads (e.g.) to enhancement of low mass dilepton production [17, 18]. However, we here obtain only a small impact on the direct photon production which is hidden below the dominant bremsstrahlung contribution. The same holds also for other in-medium scenario such as a dropping mass of the vector meson.

In Fig. 8 we specify the meson-meson bremsstrahlung by showing the contributions of different sub-channels $\pi+\pi \rightarrow \pi+\pi+\gamma, \pi+\eta \rightarrow \pi+\eta+\gamma, \pi+K \rightarrow \pi+K+\gamma$ with $K=\left(K^{+}, K^{-}\right), \pi+K^{0} \rightarrow \pi+K^{0}+\gamma, \pi+K^{*} \rightarrow \pi+K^{*}+\gamma$ and $\pi+K^{* 0} \rightarrow \pi+K^{* 0}+\gamma$. We stress again that only the elastic meson-meson collisions have been accounted here. Indeed, the final result is very sensitive to the meson-meson elastic cross section which is, however, 
very hard to measure experimentally. We have used a $10 \mathrm{mb}$ elastic cross section for all mesonmeson scatterings in the present study. As follows from Fig. 8 the dominant contribution stems from $\pi+\pi$ elastic scattering; the contributions from $\pi+K^{* 0}, \pi+K^{0}$ and $\pi+\eta$ are also visible at low $p_{T}$. This is again consistent with Refs. [27, 28] where the authors found that the photon emission rate from pion-kaon scattering is at the $\sim 20 \%$ level of the one from pion-pion scattering.

We mention that the solid line at high $p_{T}$ in Fig. 7 shows the estimated contribution from the prompt photons. For that we have convoluted the pp data fit from Ref. [44] with the nuclear overlap function $T_{A B}$. Indeed, it gives a lower estimate for the prompt photons since the nuclear effects (as e.g. Cronin effect) have been ignored. For futher discussions on the contributions of the prompt as well as thermal photons we address to Refs. [26, 44] and references therein.

\section{RESULTS FOR THE PHOTON PRODUCTION IN P+A COLLISIONS AT SPS ENERGIES}

In order to draw solid conclusions on the direct photons from heavy-ion collisions one needs some 'reference systems', i.e. to compare to $\mathrm{p}+\mathrm{p}$ and/or $\mathrm{p}+\mathrm{A}$ data. Recently the WA98 Collaboration has provided preliminary data on $\mathrm{p}+\mathrm{C}$ and $\mathrm{p}+\mathrm{Pb}$ collisions at $\sqrt{s}=17.4 \mathrm{GeV}$. In this Section we present the HSD results for these systems.

We start again with the inclusive invariant photon spectra from $\mathrm{p}+\mathrm{C}$ and $\mathrm{p}+\mathrm{Pb}$ collisions at mid-rapidity for $160 \mathrm{~A} \mathrm{GeV}$ which are depicted in Fig. 9, The solid dots corresponds to the preliminary WA98 data [19], the various lines stand for the HSD calculations. Again, as in a case of $\mathrm{Pb}+\mathrm{Pb}$, the dominant contribution comes from the $\pi^{0}$ and $\eta$ photonic decays. As seen from Fig. 9 HSD provides a very good description of the inclusive experimental data.

In order to focus on the direct photon contribution the WA98 collaboration has subtracted the contributions of the hadronic decays in a similar way as for the $\mathrm{Pb}+\mathrm{Pb}$ collisions. However, due to experimental uncertainties in the 'background ' subtraction the preliminary WA98 data correspond to the upper limits on direct photon production [19] and are shown by the (down) arrows in Fig. 10 for $\mathrm{p}+\mathrm{C}$ (upper part) and $\mathrm{p}+\mathrm{Pb}$ (lower part). The various lines in Fig. 10 stand for the HSD result. Here we do not apply any cuts in density in order to obtain an upper estimate for the hadronic decays. Contrary to the $\mathrm{Pb}+\mathrm{Pb}$ collisions the contribution from the meson-meson bremsstrahlung is suppressed compared to the $\eta^{\prime}$ and $\omega$ decay due to the small 

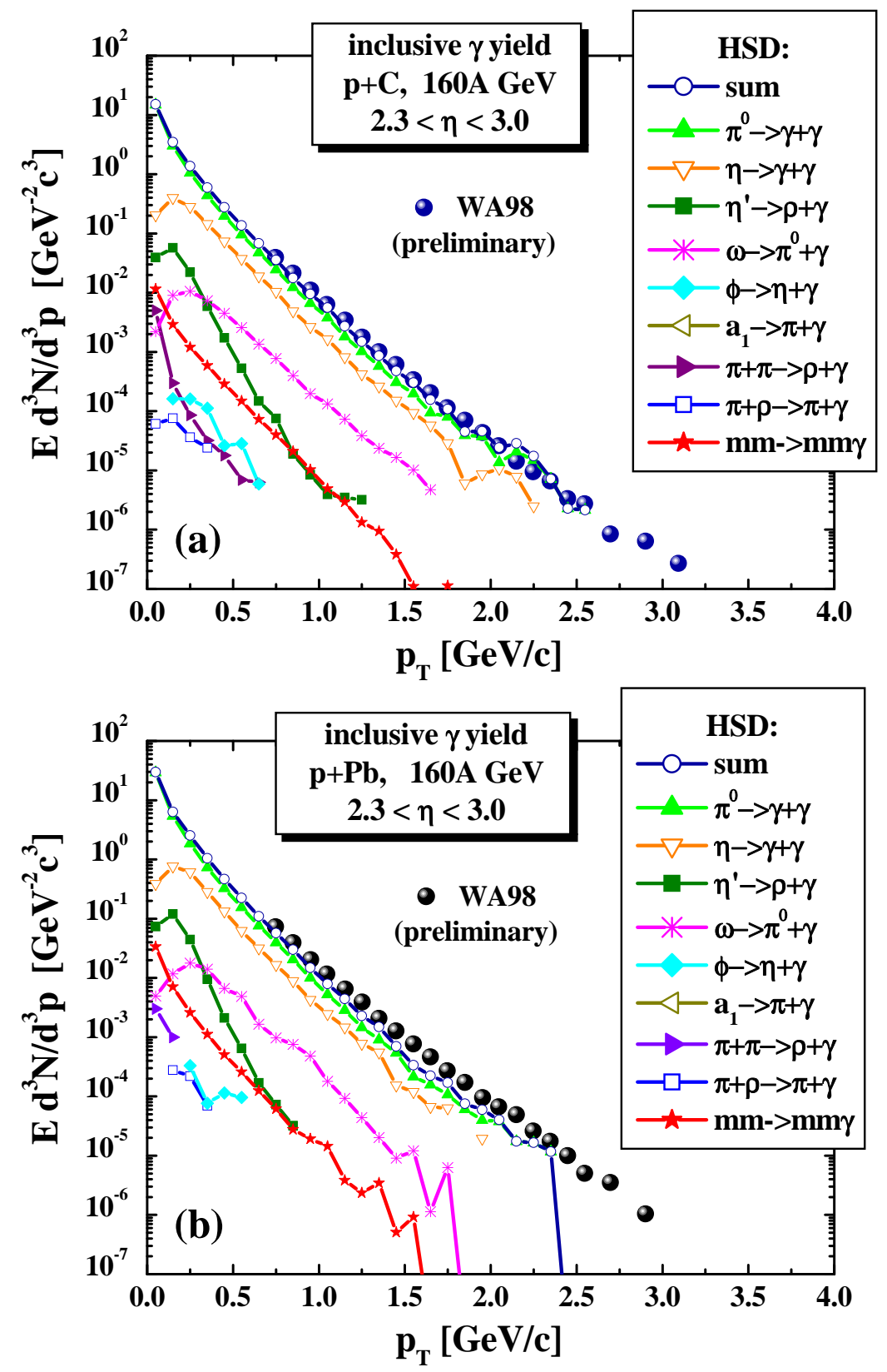

FIG. 9: (Color online) The inclusive invariant photon multiplicity for $158 \mathrm{~A} \mathrm{GeV} p+{ }^{12} \mathrm{C}$ (upper part - (a)) and $p+{ }^{208} \mathrm{~Pb}$ (lower part - (b)) at $2.3 \leq \eta \leq 3.0$. The solid dots corresponds to the preliminary WA98 data [19]; the lines stand for the HSD calculations. The definition of the lines is indicated in the legend.

meson density in $\mathrm{p}+\mathrm{A}$ collisions. As seen from Fig. 10 the HSD results are much below the upper limits from WA98. Since it is unlikely that there are some new channels for photon production in $\mathrm{p}+\mathrm{A}$ reactions, which are not visible in $\mathrm{Pb}+\mathrm{Pb}$ collisions, we expect that the 

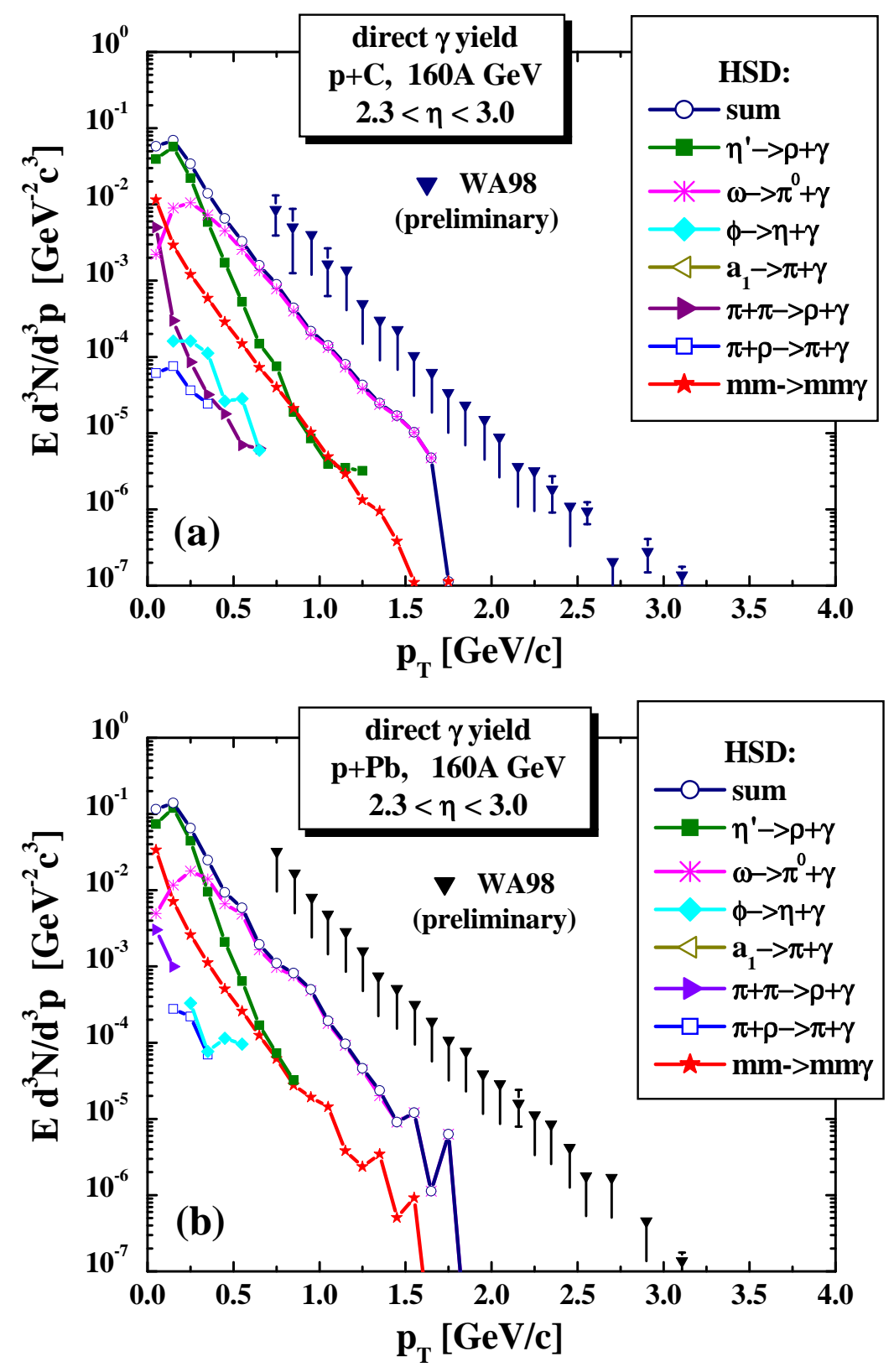

FIG. 10: (Color online) The invariant direct photon multiplicity for $158 \mathrm{~A} \mathrm{GeV} p+{ }^{12} \mathrm{C}$ (upper part (a)) and $p+{ }^{208} \mathrm{~Pb}$ (lower part - (b)) at $2.3 \leq \eta \leq 3.0$. The solid dots corresponds to the preliminary WA98 data [19]; the lines stand for the HSD calculations. The definition of the lines is indicated in the legend.

final data might go down, too. Thus, it is quite important to obtain precise $\mathrm{p}+\mathrm{A}$ data from the experimental side in order to check the theoretical models more accurately. 


\section{SUMMARY}

In this study a detailed analysis of the low $p_{T}$ photon production in $\mathrm{p}+\mathrm{C}, \mathrm{p}+\mathrm{Pb}$ and $\mathrm{Pb}+\mathrm{Pb}$ collisions at $160 \mathrm{~A} \mathrm{GeV}$ has been presented within the microscopic HSD transport approach that incorporates a full off-shell propagation of the vector mesons [17]. We stress, that the HSD approach contains only hadronic degrees of freedom and strings and doesn't include the phase transition from a QGP to hadronic matter explicitly; the partonic interactions are treated in HSD only inside the strings (and in terms of leading quarks and diquarks). However, the model has all non-equilibrium dynamical features and demonstrates a good ability in describing a variety observables for heavy-ion, proton- and pion- induced reactions. Accordingly, we apply our model to study the direct photon production from hadronic sources which dominate at low $p_{T}$.

In particular the following hadronic sources for direct photon production in heavy-ion collisions have been incorporated:

- photon emission from elementary meson-meson rescatterings, where the processes $\pi \rho \rightarrow \pi \gamma$ and $\pi \pi \rightarrow \rho \gamma$ are dominant due to the high pion production rate. The novel issue here is that we accounted for the off-shellness of the initial/final $\rho$ meson and extended the vacuum cross sections for these processes from Kapusta et al. [29] for the in-medium case with the full off-shell $\rho$ meson spectral function. It allows us to investigate the influence of the in-medium effects such as 'collisional broadening' on the photon $p_{T}$ spectrum.

- meson-meson bremsstrahlung from the elastic meson-meson scattering $m_{1}+m_{2} \rightarrow m_{1}+$ $m_{2}+\gamma\left(\right.$ where $\left.m=\pi, \eta, K, \bar{K}, K^{0}, K^{*}, \bar{K}^{*}, K^{* 0}\right)$, which we accounted for by applying the SPA formula (16) .

We have found that the enhancement of the low $p_{T}$ photon emission from elementary mesonmeson rescattering due to a collisional broadening of the vector-meson spectral functions is hardly visible in the final spectra which are dominated by bremsstrahlung type processes. Thus our non-equilibrium dynamical calculations support the early findings in Refs. [27, 28] based on the hadron gas models.

Also we have investigated the uncertainties in the extraction of the direct photon yield from the measured inclusive photon spectra which are dominated by the hadronic decays, in particular $\pi^{0}, \eta, \eta^{\prime}, \omega$ and $a_{1}$. Here the HSD model shows a very good agreement with the measured inclusive photon transverse momentum distribution. While the 'background' 
contributions from the $\pi^{0}$ and $\eta$ decay can be subtracted in a reliable way - since these mesons are measured by the same WA98 experiment - there remains a problem in the subtraction of the contributions from short living resonances which decay inside the hot and dense fireball. We have estimated the possible contribution of such in-medium decay processes by comparing two calculations - in the first we have selected the photons coming from the mesonic decays inside the fireball by applying a density cut $\rho_{N} \geq \rho_{0} / 2$, in the second we have accounted for the mesonic decays over the full time history. We found that the density cut reduces drastically the final yield which depends directly on the actual value of the cut. However, some amount of photons from the inside $\omega$ decay for $\rho_{N} \geq \rho_{0} / 2$ is visible in the final spectra and might be misidentified as direct photons.

For reference we have also studied the photon production for $\mathrm{p}+\mathrm{C}$ and $\mathrm{p}+\mathrm{Pb}$ collisions at $160 \mathrm{GeV}$. Again the HSD model provides a good description of the preliminary experimental data from the WA98 Collaboration on the inclusive spectra, however, is much below the upper

limits for the direct photons (even without a subtraction of $\eta^{\prime}, \omega, \phi$ and $a_{1}$ photon decays). In this respect it will be very helpful to have the final experimental data (with high accuracy).

\section{Acknowledgments}

The authors acknowledge inspiring discussions with W. Cassing, C. Gale, R. Rapp and S. Turbide. This work was partially supported by INTAS, grant number 06-1000012-8914, and the Federal agency of Russia for atomic energy (Rosatom).

[1] E.L. Feinberg, Nuovo Cimento 34 A, 391 (1976).

[2] E. Shuryak, Phys. Lett. B 78, 150 (1978).

[3] J.D. Bjorken and H. Weisberg, Phys. Rev. D 13, 1405 (1976).

[4] R.D. Pisarski, Phys. Rev. Lett. 63, 1129 (1989).

[5] E. Braaten, R.D. Pisarski, and T.C. Yuan, Phys. Rev. Lett. 64, 2242 (1990).

[6] J. Kapusta, P. Lichard, and D. Seibert, Phys. Rev. D 44, 2774 (1991); Erratum, ibid D 47, 4171 (1993).

[7] D. K. Srivastava, Eur. Phys. J. C 10, 487 (1999). 
[8] Z. W. Lin et al., Nucl. Phys. A 698, 375 (2002).

[9] S.A. Bass and A. Dumitru, Phys. Rev. C 61, 064909 (2000).

[10] W. Cassing, Nucl. Phys. A 791, 365 (2007); ibid A 795, 70 (2007).

[11] R. Albrecht et al., WA98 Collaboration, Phys. Rev. Lett. 76, 3506 (1996).

[12] A. Dumitru, U. Katscher, J. A. Maruhn, H. Stöcker, W. Greiner, and D. H. Rischke, Phys. Rev. C 51, 2166 (1995).

[13] G.Q. Li, G.E. Brown, C. Gale, and C.M. Ko, nucl-th/9712048.

[14] E. L. Bratkovskaya, W. Cassing, Nucl. Phys. A 619, 413 (1997).

[15] M.M. Aggarval et al., WA98 Collaboration, nucl-ex/0006007; Phys. Rev. Lett. 85, 3595 (2000); ibid 93, 022301 (2004).

[16] T. Peitzmann and M.H. Thoma, Phys. Reports 364, 175 (2002).

[17] E.L. Bratkovskaya and W. Cassing, Nucl. Phys. A 807, 214 (2008).

[18] E. L. Bratkovskaya, W. Cassing, and O. Linnyk, arXiv:0805.3177 [nucl-th].

[19] C. Baumann et al., WA98 Collaboration, arXiv:0804.4407 [nucl-ex].

[20] S.M. Kiselev, arXiv:0701130 [hep-ph].

[21] W. Cassing and E. L. Bratkovskaya, Phys. Rept. 308, 65 (1999).

[22] W. Ehehalt and W. Cassing, Nucl. Phys. A 602, 449 (1996).

[23] K. Weber et al., Nucl. Phys. A 539, 713 (1992).

[24] B. Anderson, G. Gustafson, and Hong Pi, Z. Phys. C 57, 485 (1993).

[25] Particle Data Group, J. Phys. G 33, 1 (2006).

[26] S. Turbide, R. Rapp, and C. Gale, Phys. Rev. C 69, 014903 (2004).

[27] K. Haglin, J. Phys. G 30, L27 (2004).

[28] W. Liu and R. Rapp, Nucl. Phys. A 96, 101 (2007).

[29] J. Kapusta et al., Phys. Rev. D 44, 2774 (1991).

[30] T. Hatsuda and S. Lee, Phys. Rev. C 46, R34 (1992).

[31] G.E. Brown and M. Rho, Phys. Rev. Lett. 66, 2720 (1991).

[32] R. Rapp, G. Chanfray, and J. Wambach, Phys. Rev. Lett. 76, 368 (1996).

[33] V. Metag, arXiv:0711.4709 [nucl-ex].

[34] K. Haglin, Nucl. Phys. A 584, 719 (1995).

[35] D. Trnka et al., Phys. Rev. Lett. 94, 192303 (2005). 
[36] M. Zielinski et al., Phys. Rev. Lett. 52, 1195 (1984).

[37] V. Koch, Int. J. Mod. Phys. E 6, 203 (1997).

[38] R. Rapp, J. Phys. G 31, S217 (2005).

[39] W. Wagner and S. Leupold, arXiv:0708.2223 [hep-ph].

[40] S. Vogel and M. Bleicher, arXiv:0710.1158 [hep-ph].

[41] C. Gale and J. Kapusta, Phys. Rev. C 35, 2107 (1987); C. Gale and J. Kapusta, Nucl. Phys. A 495, 423c (1989).

[42] E. L. Bratkovskaya et al. Phys. Rev. C 69, 054907 (2004).

[43] W. Cassing, E. L. Bratkovskaya, and A Sibirtsev, Nucl. Phys. A 691, 753 (2001).

[44] D.K. Srivastava, Eur. Phys. J. C22, 129 (2001). 